Objectives: We have investigated the hypothesis that statin, an agent with pleiotropic effects, could augment the therapeutic potential of AdSCs. Methods: ILD was induced by bleomycin (BLM) in C57BL/6 mouse, and the mice were assigned in the following groups: 1) Control, 2) NP-AdSCs $\left(2.5 \times 10^{4}\right.$ cells), and 3$)$ STNP-AdSCs $\left(2.5 \times 10^{4}\right.$ cells $)$.

Results: Simvastatin-conjugated nanoparticles (STNP) significantly promoted the migration activity and cell survival without changing the proliferation activity, and up-regulated transforming growth factor (TGF) $-\beta 1$ in vitro assays. Lung inflammation and fibrosis assessed were significantly supressed at 4 weeks after starting BLM administration in STNP-AdSCs group (Figure). The levels of IL-4, IFN-gamma, TNF-alpha, COL1A1, and TIMP1 mRNA expression at 28 days after BLM administration were significantly lower in STNP-AdSCs group compared with that in other groups. Conclusion: Simvastatin-conjugated nanoparticles enhanced the therapeutic effect of a small number of AdSCs transplantation.
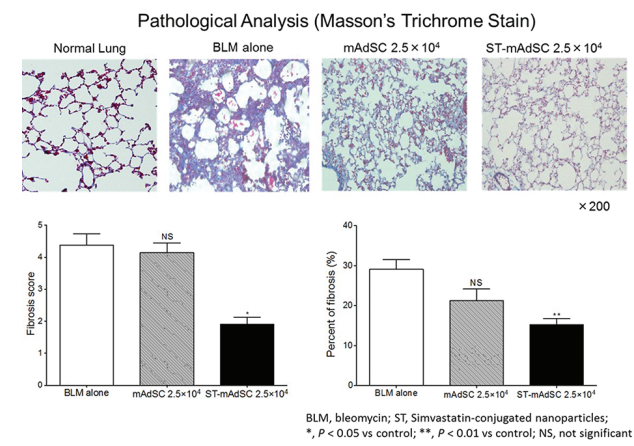

Abstract THU0338 - Figure 1

REFERENCE:

[1] Sci Rep 6; 7 (1): 14608, 2017.

Acknowledgement: This study was supported by Grants-in-Aid for Scientific Research-KAKENHI- of Japan (18K08160).

Disclosure of Interests: None declared

DOI: 10.1136/annrheumdis-2019-eular.6636

\section{THU0339 PULMONARY INVOLVEMENT AND OUTCOME IN SYSTEMIC SCLEROSIS (SSC) - ILD-PH AS AN IMPORTANT SUBSET}

Michael Kreuter ${ }^{1}$, Francesco Bonella ${ }^{2}$, Ulf Müller-Ladner ${ }^{3}$, Elise Siegert ${ }^{4}$ Jörg Henes ${ }^{5}$, Gabriela Riemekasten ${ }^{6}$, Norbert Blank ${ }^{7}$, Marc Schmalzing ${ }^{8}$, Ina Koetter ${ }^{9}$, Claudia Guenther ${ }^{10}$, Tim Schmeiser ${ }^{11}$, Gabriele Zeidler ${ }^{12}$, Alexander Kreuter ${ }^{13}$, Margitta Worm ${ }^{14}$, Laura Susok ${ }^{15}$, Aaron Juche ${ }^{16}$, Christiane Pfeiffer ${ }^{17}$, Cord Sunderkoetter ${ }^{18}$, Donja Homayoon ${ }^{19}$, Kathrin Kuhr ${ }^{20}$, Hanns-Martin Lorenz ${ }^{21}$, Pia Moinzadeh ${ }^{22}$, Nicolas Hunzelmann ${ }^{22} .{ }^{1}$ Center for interstitial and rare lung disease, Thoraxklinik, University Hospital, Pneumology, Heidelberg, Germany; ${ }^{2}$ Center for interstitial and rare lung disease Ruhrlandklinik University Hospital, Essen, Germany; ${ }^{3}$ Justus Liebig University, Campus Kerckhoff, Rheumatology, Giessen, Germany; ${ }^{4}$ Charité - Universitätsmedizin Berlin, Rheumatology, Berlin, Germany, ${ }^{5}$ Center for Interdisciplinary Clinical Immonology, Rheumatology and Autoinflammatory Diseases, University Hospital, Tuebingen, Germany; ${ }^{6}$ University Medical Center - UKSH, Rheumatology, Luebeck, Germany, ${ }^{7}$ University Hospital Heidelberg, Rheumatology, Heidelberg, Germany; ${ }^{8}$ University Hospital Würzburg, Rheumatology, Würzburg, Germany; ${ }^{9}$ Asklepios Clinik Altona, Internal Medicine, Rheumatology, Immunology and Nephrology, Hamburg, Germany, ${ }^{10}$ University Hospital Carl Gustav Carus, Dermatology, Dresden, Germany, ${ }^{11}$ Hospital St. Joseph, Rheumatology, Wuppertal, Germany, ${ }^{12}$ Johanniter-Krankenhaus im Fläming, Rheumatology, Treuenbrietzen, Germany; ${ }^{13} \mathrm{HELIOS}$ St. Elisabeth Hospital Oberhausen, University Witten-Herdecke, Dermatology, Oberhausen, Germany, ${ }^{14}$ Charité Universitätsmedizin Berlin, Dermatology, Berlin, Germany; ${ }^{15}$ Ruhr-UniversityBochum, St. Josef Hospital, Dermatology, Bochum, Germany, ${ }^{16}$ Immanuel Hospital Berlin (Buch), Rheumatology, Berlin, Germany, ${ }^{17}$ University Hospital Ulm, Dermatology, Ulm, Germany, ${ }^{18}$ University Hospital Halle (Saale), Dermatology, Halle, Germany; ${ }^{19}$ Medical University of Graz, Dermatology, Graz, Austria; ${ }^{20}$ University of Cologne, IMSB, Cologne, Germany; ${ }^{21}$ Center for interstitial and rare lung disease Ruhrlandklinik University Hospital, Pneumology, Essen, Germany; ${ }^{22}$ University Hospital Cologne, Dermatology, Cologne, Germany

Background: Pulmonary involvement is the leading cause of death in SSc and can manifest as interstitial lung disease (ILD), pulmonary hypertension (PAH) or a combination (ILD-PH). Aim of this analysis was to determine prevalence, clinical characteristics and outcomes of different forms within the German SSc Network.

Objectives:

Methods: SSc patients were analyzed for pulmonary involvement, clinical characteristics and outcome.

Results: There were 3699 pts in 42 centers with a mean follow up time of $34.4 \pm 12.6$ months. At baseline, ILD was frequent $(29.5 \%)$, while ILD $\mathrm{PH}$ and $\mathrm{PAH}$ had lower prevalences $(7.5 \%, 6.1 \%)$. At the end of follow up, $32 \%$ of SSc pts had ILD, $13 \%$ ILD-PH and $7 \%$ PAH. ILD and ILD $\mathrm{PH}$ were more frequent in the diffuse form $(47 \%, 12 \%)$, while $\mathrm{PAH}$ did not differ between subforms. Significant differences in baseline characteristics between PAH vs. ILD-PH vs. ILD were found for age (62, 59, 54 years), sex (males: 15\%, 22\%, 24\%) and smoking prevalence (non-smok ers $49 \%, 63 \%, 57 \%$ ). Mean DLCO and FVC were $56 \% / 93 \%$ for PAH, $49 \% / 78 \%$ for ILD-PH and $56 \% / 81 \%$ for ILD. Significant decreases for DLCO $(\geq 15 \%)$ and FVC $(\geq 10 \%)$ were found in $45 \% / 26 \%$ in $\mathrm{PAH}, 45 \% /$ $26 \%$ for ILD-PH and $36 \% / 16 \%$ in ILD. All-cause mortality was $8.1 \%$ for the total cohort and differed significantly between patients without pulmo nary involvement (4\%), ILD (7.8\%), PAH (14.2\%), and ILD-PH (21\%, $\mathrm{p}<0.001$ ).

Conclusion: ILD is the most prevalent pulmonary involvement in SSc, while PH-ILD is associated with the most detrimental survival. Significant differences in baseline characteristics of types of pulmonary SSc involvement may help to identify patients at risk in the future.

Disclosure of Interests: : Michael Kreuter Grant/research support from: Boehringer Ingelheim, Roche Pharma, Consultant for: Boehringer Ingelheim, Roche Pharma, Speakers bureau: Boehringer Ingelheim, Roche Pharma, Francesco Bonella Consultant for: Boehringer Ingelheim, Roche Pharma, Speakers bureau: Boehringer Ingelheim, Roche Pharma, Ulf Müller-Ladner Grant/research support from: Projekt supported by an unrestricted educational grant from Celgene $\mathrm{GmbH}$., Elise Siegert Shareholder of: Astra Zeneca, Grant/research support from: Actelion, Consultant for: AEC Partners, Speakers bureau: Actelion, Norsk, Jörg Henes: None declared, Gabriela Riemekasten Consultant for: Chugai, F. Hoffmann-La Roche, Speakers bureau: Chugai, F. Hoffmann-La Roche, Norbert Blank Grant/research support from: SOBI and Novartis, Speakers bureau: Novartis and SOBI, Marc Schmalzing Grant/research support from: Pfizer, Chugai, MSD, Janssen-Cilag, BMS, Celgene, UCB, Consultant for: Abbvie, Chugai, Genzyme, Hexal/Sandoz, MSD, Novartis, Roche, Sanofi Pasteur Speakers bureau: Actelion, Baxalta/Shire, BMS, Celgene, Chugai, Janssen-Cilag, MSD, Novartis, Pfizer, Roche, UCB, Ina Koetter: None declared, Claudia Guenther Grant/research support from: Pfizer, Novartis, Employee of: 20 years ago, Novartis, Speakers bureau: Celtis, Tim Schmeiser Speakers bureau: Actelion, UCB, Pfizer, Gabriele Zeidler Grant/research support from:

- MSD Sharp \& Dohme GmbH

- Actelion Pharmaceuticals Deutschland GmbH

- Roche Pharma AG

- AbbVie Deutschland GmbH Co. KG

- Pfizer Pharma GmbH

- Lilly Deutschland GmbH

- Celgene $\mathrm{GmbH}$

Bristol-Myers Squibb

UCB Pharma GmbH/UCB GmbH, Speakers bureau:

- MSD Sharp \& Dohme GmbH

- Actelion Pharmaceuticals Deutschland $\mathrm{GmbH}$

- Roche Pharma AG

- AbbVie Deutschland GmbH Co. KG

- Pfizer Pharma GmbH

- Lilly Deutschland GmbH

- Celgene $\mathrm{GmbH}$

\section{Bristol-Myers Squibb}

UCB Pharma GmbH/UCB GmbH, Alexander Kreuter Speakers bureau: Actelion Pharma, MSD, AbbVie, InfektioPharm, Margitta Worm: None declared, Laura Susok: None declared, Aaron Juche: None declared, Christiane Pfeiffer: None declared, Cord Sunderkoetter: None declared Donja Homayoon: None declared, Kathrin Kuhr: None declared, HannsMartin Lorenz: None declared, Pia Moinzadeh Speakers bureau: Actelion, Nicolas Hunzelmann Speakers bureau: Boehringer Ingelheim, Actelion, Pfizer, Roche

DOI: 10.1136/annrheumdis-2019-eular.1775 